\title{
APPLICATIONS OF AUTOSTEREOSCOPIC DISPLAYS IN OPHTHALMOLOGIC STUDIES
}

\author{
Xiaoyi Jiang ${ }^{1}$ and Daniel Mojon ${ }^{2}$ \\ ${ }^{1}$ Department of Mathematics and Computer Science, University of Münster, Germany \\ Email: xjiang@math.uni-muenster.de \\ ${ }^{2}$ Kantonsspital St. Gallen, Department of Neuro-Ophthalmology, Switzerland \\ Email: daniel.mojon@kssg.ch
}

\begin{abstract}
Autostereoscopic displays are an emerging technology which provide 3D viewing experiences without the need of glasses or other encumbering viewing aids. In this paper we explore their potential in ophthalmologic studies. While the vast majority of applications of autostereoscopic displays in other fields is merely based on producing $3 \mathrm{D}$ viewing effects, we can distinguish between several classes of ophthalmologic tasks in which autostereoscopic displays play a very different role. Three concrete applications are described. With the steady improvements in autostereoscopic displays we expect to develop qualitatively new ophthalmologic tests in future.
\end{abstract}

\section{INTRODUCTION}

As a discipline of investigating the human's visual perception and disorders, ophthalmology is full of visual tests. They help conduct basic and clinic studies for visual phenomena, make medical diagnoses, and measure the sensory outcomes after treatment. Currently, these tests are typically done by means of cards. For instance, the standard for testing random-dot stereovision under natural conditions is the Lang Test. It is similar to 3D postcards and shows several objects (car, cat, etc.) above a random background in depth. The test person has to say what he/she sees.

For various reasons these tests are at best suboptimal. All the cards are static by nature and thus cannot be adapted in terms of the number of figures. the figures themselves, and their positions. In addition verbal communication is the basic assumption so that it is hardly possible to use them for preverbal children or other subjects with difficulties in verbal communication.

In this work we describe our work of designing screenbased visual tests using autostereoscopic displays towards overcoming the drawbacks of previous tests. We start with a short description of autostereoscopic displays. Then, some general thoughts to applying autostereoscopic displays in ophthalmologic studies are given, followed by details of several tests we have realized so far. Finally, some discussions conclude the paper.

\section{AUTOSTEREOSCOPIC DISPLAYS}

Autostereoscopic displays provide 3D viewing experiences without the need of glasses or other encumbering viewing aids ${ }^{1}$. They are appealing because the users obtain the best approximation of natural working in a 3D computer environment. The basic function of such a display is to distribute the left and right view of a scene to the corresponding eye of the viewer independently. Then, the human visual system processes the stereo image pair and the viewer will perceive depth.

One popular approach to autostereoscopic display is the lenticular sheet technique. A lenticular sheet consists of a series of semi-cylindrical vertical lenses. Each lens focuses on the image information located behind it and directs the light in different directions. A stereo image pair is displayed by interleaving columns of the two images, one column of each image per column of lenses. Parallax barrier is another widely used principle of autostereoscopic displays. Such a system consists of a series of fine vertical slits in an otherwise opaque medium. The stereo image pair is again displayed in an interleaving manner. If the interleaved images are properly positioned behind a back lit barrier and the viewer is the required distance from the barrier, then the barrier will occlude the appropriate image columns to the eyes and the viewer will perceive an autostereoscopic image.

The interested readers are referred to $[5,6,8]$ for an overview of autostereoscopic display techniques and to [10, 12] for a summary of (mostly commercially available) autostereoscopic displays. Besides 3D computer games, several other applications have been suggested, for instance human-computer interface [9] and computer-assisted microsurgery [4]. Our focus are ophthalmologic studies. In our work we have used a 2018XLQ 19" autostereoscopic display from DTI [11] which is based on the parallax barrier principle. It has 1280 columns and 1024 rows of pixels, resulting in a stereoscopic image pair of resolution $640 \times 1024$ each. The best 3D viewing quality

\footnotetext{
${ }^{1}$ In the three-dimensional display field, the term autostereoscopic has two different meanings: the narrow one restricted to one single screen (also called parallax displays) and the broad one, in which auxiliary devices like mirrors are needed. The current work only considers the first one.
} 
is perceived at a certain distance which is dependent of the interpupillary distance of the observer and can be calculated trigonometrically.

\section{TAXONOMY OF USING AUTOSTEREOSCOPIC DISPLAYS IN OPHTHALMOLOGY}

Autostereoscopic displays have been becoming popular for a variety of applications. They share all the fact that the intention is simply to produce 3D effects to be seen by users.

The situation in ophthalmology is much more multifarious. The potential applications can be classified according to two different criteria. We may distinguish between different applications based on the test person:

- He/she has full 3D perception function. The goal is to investigate his/her behavior in a 3D environment. This kind of tests is in line with the vast majority of applications in other fields, where autostereoscopic displays serve for producing $3 \mathrm{D}$ viewing effects.

- It is unknown if he/she has full 3D perception function. As a matter of fact, the task of such tests is to make a diagnosis on the person's 3D perception function.

Alternatively, the second criterion is whether a meaningful stereo image pair should be displayed. There are many ophthalmological tests concerned with one eye only. This is usually achieved by covering the other eye, for instance by a paper sheet. In this case we can simply compose a "stereo" image pair from the test image and another contentless image (of constant intensity/color). The use of an autostereoscopic display would enable the same visual test under more natural testing conditions. In the following we describe several applications we have realized so far. They cover the full spectrum of this taxonomy.

\section{INFLUENCE OF PULFRICH PHENOMENON ON DRIVING PERFORMANCE}

In 1922, Pulfrich reported that with a neutral density filter in front of one eye the path of a swinging pendulum moving in a frontal plane appears to be elliptical in depth. The most generally accepted explanation for this stereo illusion is that reducing the luminosity to one eye causes a delayed processing of the image relative to the other eye. As a consequence, information about scenes imaged on the two retinas at different times arrive simultaneously at the cortex (spatial disparity), or information about scenes imaged on the two retinas at the same time arrives asynchronously at the cortex (temporal disparity). In such a situation, a moving object is perceived at a wrong distance. Data from several psychophysical and electrophysiological investigations are consistent with the delay hypothesis. In addition, several diseases induce asymmetric delays in the visual pathway and the stereo illusion can also
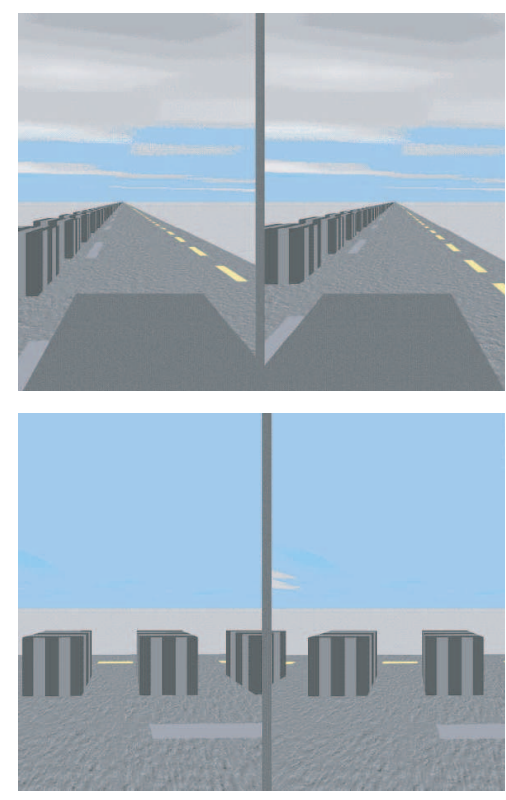

Fig. 1. Computer-generated driving scenery. Note that here the left and right image are shown in a side-by-side mode. This is one of the input formats accepted by the DTI autostereoscopic display. Its electronics automatically transforms this input into the final interleaving format in real time.

be perceived there without a filter. In such cases the term spontaneous Pulfrich phenomenon is used.

Forty years ago, a traffic accident secondary to a Pulfrich phenomenon induced by unilateral papillary dilatation has been reported. Since then, several authors reported that patients harboring a spontaneous Pulfrich phenomenon claim to have difficulties while driving. However, no study has ever examined the risk of driving of patients harboring a Pulfrich phenomenon. In a recent study [2] we determined influence of the Pulfrich phenomenon on driving performance using a computer driving simulation.

Our simulation created stereo pairs of images in real time. The scenery simulated driving on a straight road with a wide plain space on each side. The car was visualized on the screen with the fender. On the left side of the car, cubic obstacles were visible. Relative to the road, these obstacles were not moving. The task of the driver consisted in driving in such a way that a vertical bar attached to the left side of the car was as close as possible to the right surface of the cubes. Figure 1 shows two examples of angle of view $45 \%$ and $90 \%$, respectively. The participants drove the same driving scenery under different conditions (angles of view, speeds, and time delay of one eye to the other). The software recorded the position of the car during each experiment. Multivariate statistical analysis has been done to analyze the outcome variable of distance between the bar (representing the car position) and the obstacles as a function of driving conditions. 
In this application the autostereoscopic display serves for producing $3 \mathrm{D}$ viewing effects. We have made the experience that natural viewing conditions is extremely important for 3D simulation. As a matter of fact, we started the simulation by using a normal 2D screen (side-by-side display mode) and prisms allowing the fusion of the stereo images. However, it turned out that most of the test persons had considerable difficulties in fusing the images this way. After switching to the autostereoscopic display, we did not encounter any real trouble in conducting the experiments.

\section{RANDOM-DOT STEREOTEST}

Over the past 25 years, many psychophysical and electrophysiological stereotests have been developed for infants. While the main interest of earlier investigations was to study the development of stereopsis, several later studies focused on the measurement of sensory outcomes following the treatment of ophthalmopediatric disorders. Many testing procedures use random-dot stereograms since they convey no visual information other than random noise, if seen monocularly. However, if binocularly fused, vivid depth perceptions occur. This lack of monocular cues makes this type of stereograms ideal for stereovision testing.

In clinical routine, the most frequently used random-dot tests include the TNO Test, the Lang Test, and the Randomdot E Test. All these tests need verbal capabilities of the subject tested. Tests applicable to preverbal children are the Infant Random Dot Stereoacuity Cards, random-dot stereogram evoked potentials, and random-dot correlogram evoked potentials. These approaches, however, suffer from the need of special glasses and other difficulties [3,7].

For screening of visual dysfunctions in children and for large scale testing of the development of stereovision an objective test easy to perform should be available. We developed a new, 3D-monitor based random-dot test allowing natural viewing conditions with an objective assessment of stimulus recognition $[3,7]$.

The experimental setup for children older than 1.5 years is sketched in Figure 2 (left); see Figure 3 for a picture of this setup. The children were seated on the lap of the mother or alone, with their heads stabilized by a chin and front rest. The monitor for stimulus presentation was placed in a frontoparallel position at the optimal distance from the eyes. An infrared illumination of the eye $(880 \mathrm{~nm})$ was used to produce the corneal reflex and the pupil image. The illumination source and camera were installed above the children's head. A hot mirror (dichroic filter separating visible light and infrared light) was used to illuminate the eye and to register the reflexes with the camera. Another experimental setup is used for children up to 1.5 years, see Figure 2 (right).

A visual stimulus, for instance circle, is placed with some distance above a plane. Both the stimulus and this plane are coded by a random-dot pattern such that no monocular cues
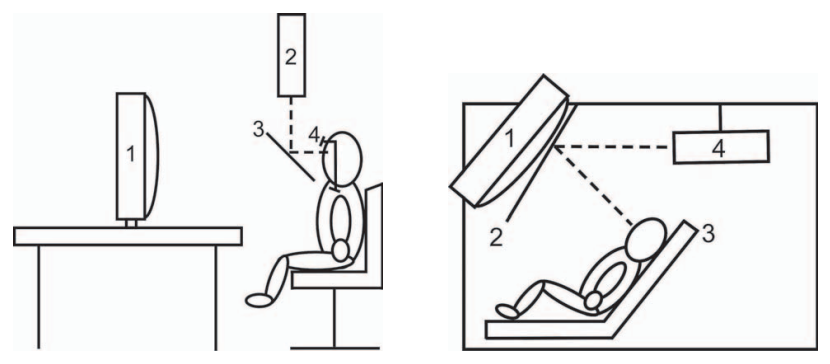

Fig. 2. Experimental setting of random-dot stereotest for children older than 1.5 years (left): $1=$ autostereoscopic display for stimulus presentation; $2=$ infrared illumination and infrared photo-oculography camera; $3=$ hot mirror; $4=$ chin and front rest. Experimental setting for children up to the age of 1.5 years (right): $1=$ autostereoscopic display for stimulus presentation; $2=$ hot mirror; $3=$ infant car seat; $4=$ infrared illumination and camera.

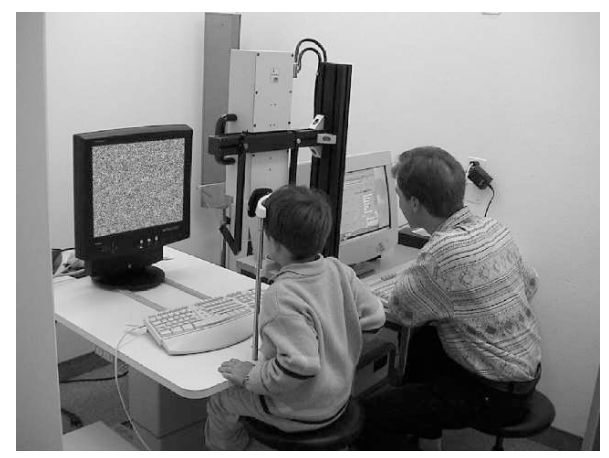

Fig. 3. Experimental setting of experimental setting of random-dot stereotest for children older than 1.5 years.

are visible. An experimental session shows the stimulus at different known positions at known speed. An infrared photooculography system was used to record the eye movements. The recorded eye movement is compared to the expected eye movements if all stimulus positions are perfectly followed by the test person. Then, we determined in a masked manner, without knowledge of the diagnosis and stereoscopic functions, which saccade ends corresponded to the presented stimulus positions. A positive response was assumed if during the whole registration period two or more consecutive saccades corresponded to the stimulus coordinates. Figure 4 shows such a pair of recorded eye movement and the expectation. In this case there is an excellent correspondence of eye positions with the stimulus position for both axes. Therefore, the stimulus has been recognized during nearly the whole registration period.

This random-dot stereotest represents an application situation, in which not the pure 3D effect is needed for people with full stereoscopic function. Instead, it is the goal of this test to verify the function and eventually to provide a quantitative measure of the function. 


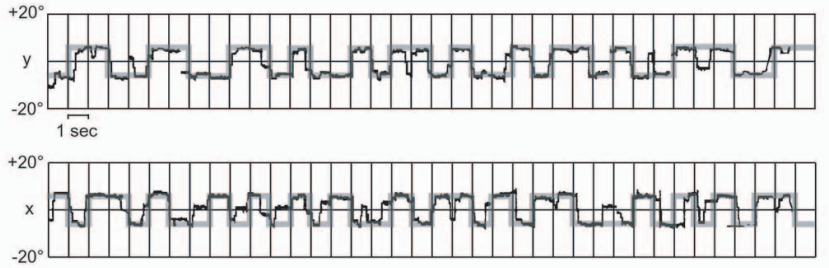

Fig. 4. Eye movement recording of a normal, 5 years old child. The ordinate labeled by $x$ corresponds to the horizontal eye positions (degrees), the ordinate labeled $y$ to the vertical ones, both plotted against time (seconds). The black line corresponds to the recorded eye positions, the gray, transparent one to the positions of the stimulus.

\section{SINGLE-EYE TESTS}

The preferential looking test using Teller acuity cards is widely used for preverbal children. Some weaknesses of this approach has been long recognized; in particular it is examiner dependent and at best half-objective. This test is a representative of a class of tests where only one eye is involved. To realize this kind of tests on a display we are faced with the fundamental trouble of covering the other eye. While this requirement could be achieved by, for instance, patching one eye, it provokes an unnatural testing environment. Ideally, we like to manage it done without such auxiliary means.

In this case autostereoscopic displays give us a perfect device for achieving our goal. We can simply assign one image channel for the test images and leave the other channel unused. That is, we compose a "stereo" image pair from the test image and another contentless image (of constant intensity/color). Showing such an image stream on an autostereoscopic display would enable the same visual test under more natural testing conditions. This general principle is basically applicable to all single-eye tests as far as the test itself can be simulated on a 2D display with a covered eye. We have applied this principle to the preferential looking test; see [1] for details.

\section{DISCUSSIONS}

The discussion on the taxonomy of using autostereoscopic displays in Section 3 reveals their potential in ophthalmology. The first application as exemplified in the Pulfrich simulation is quite straightforward and in line with a vast majority of tasks in other fields, where autostereoscopic displays merely serve for producing $3 \mathrm{D}$ viewing effects. The other application possibilities either enable us to verify the $3 \mathrm{D}$ perception function of test persons such as random-dot stereotest or to conduct arbitrary single-eye tests. Compared to other applications reported in the literature, these two latter cases represent some unique aspects of ophthalmologic studies and novel applications of autostereoscopic displays.
We have implemented several applications as outlined in this paper and further studies will be done in future. The steady progress in autostereoscopic displays opens the door to qualitatively new ophthalmologic tests. The random-dot stereotest, for instance, gives us a more reasonable way of visual communication with preverbal children or any nonverbal subjects for whatever reason who are unable to understand psychophysical instructions and to respond verbally. In addition single-eye tests can be realized in order to obtain a more natural working environment.

\section{Acknowledgments}

The work was partly supported by the Stiftung OPOS Zugunsten von Wahrnehmungsbehinderten, St. Gallen, Switzerland.

\section{REFERENCES}

[1] A. Breyer, X. Jiang, A. Rütsche, and D. Mojon, A new objective visual acuity test: An automated preferential looking. Klinische Monatsblätter Augenheilkunde, 220: 93-95, 2003.

[2] A. Breyer, X. Jiang, A. Rütsche, H. Bieri, T. Oexl, A. Baumann, and D.S. Mojon, Influence of the Pulfrich phenomenon on driving performance, Graefes Archive for Clinical and Experimental Ophthalmology, 2006. (accepted for publication)

[3] A. Breyer, X. Jiang, A. Rütsche, and D.S. Mojon, A new 3D monitor based random-dot stereotest for children, 2006. (submitted for publication)

[4] P. Chios and A.D. Linney, The design process of an autostereoscopic viewing interface for computer-assisted microsurgery, Proc. of IEEE Int. Conf. on Systems, Man and Cybernetics, Vol. 1, 136-143, 2004.

[5] N.A. Dodgson, Autostereoscopic 3D displays, Computer, 38(8), 31-36, 2005.

[6] M. Halle, Autostereoscopic displays and computer graphics, Computer Graphics, ACM SIGGRAPH, 31(2): 58 62, 1997.

[7] B. Kriegbaum-Stehberger, X. Jiang, and D.S. Mojon, Performance of a new, 3D monitor based random-dot stereotest for children aged 2 month to 6 years, 2006. (in preparation)

[8] D.F. McAllister, 3D displays, Encyclopedia on Imaging, Wiley, 1327-1344, 2002.

[9] S. Pastoor, J. Liu, and S. Renault, An experimental multimedia system allowing 3-D visualization and eyecontrolled interaction without user-worn devices, IEEE Transactions on Multimedia, 1(1): 41-52, 1999.

[10] http://www.3dcgi.com/cooltech/displays/displays.htm.

[11] http://www.dti3d.com.

[12] http://www.stereo3d.com/displays.htm 